\title{
Adaptive Filter using Improved Pigeon Inspired Optimization Algorithm for Satellite Image Denoising
}

\author{
Thomas Thangam \\ Lecturer, International Maritime College of Oman, \\ Sohar, Sultanate of Oman \\ thomasthangam13@gmail.com
}

\begin{abstract}
Satellite imaging is a current development in image processing; however, it faces a lot of challenges because of the environmental factors. For denoising, state-of-the-art method has developed some filters like the hyperspectral satellite images, which is not effectual. Moreover, this paper proposed an adaptive filter using the assist of an optimization approach for the satellite image denoising. The developed adaptive filter performs the image denoising via noise correction, noise identification, and image enhancement. In the satellite image by transforming the image to a binary image, the type- 2 fuzzy filter recognizes the noisy pixels which are passed via the adaptive non-local mean filter for the noise correction. Subsequently, the kernel-based interpolation scheme performs the image enhancement, which is performed through the developed improved Pigeon optimization algorithm (IPOA). The whole experimentation of the developed denoising system is performed taking into consideration by satellite images from standard databases. It is obvious that the developed adaptive filter with the developed improved Pigeon optimization algorithm has enhanced performance with the PSNR values from the outcomes.
\end{abstract}

Keywords: Image Processing; Denoising; Fuzzy Filter; Kernel; Optimization Algorithm

\begin{tabular}{ll} 
Nomenclature & \\
\hline Abbreviations & Descriptions \\
\hline ANLMF & Adaptive Non-Local Mean Filtering \\
PSNR & Peak Signal Noise Ratio \\
ACS & Adaptive Cuckoo Search \\
BDCS & Bandelet Denoising Compressed Sensing \\
PSO & Particle Swarm Optimization \\
IBT & Iterative Bandelet Thresholding \\
CS & Cuckoo Search \\
SSIM & Structural Similarity Index Measure \\
NLMF & Non-Local Means Filter \\
GSTV-SC & Group Sparse Total Variation with Stepsize Constraints \\
GA & Genetic Algorithm \\
CNN & Convolutional Neural Network \\
NDVI & Normalized Difference Vegetation Index \\
WF & Weiner Filter \\
DA & Dragonfly Algorithm \\
NIR & Near-Infrared Band \\
PIO & Pigeon Inspired Optimization \\
\hline
\end{tabular}

\section{Introduction}

In the present time, satellite images encompass an overabundance of applications predominantly in the areas of weather forecasting, oceanographic studies, forestry, and agriculture, planning and intelligence and so on. The edges or the high-frequency components, in attendance in those images comprise a very important part of the information. Regrettably, owing to the image acquisition lacking and transmission systems, the images acquire gets worsen by noise. Consequently, there arise awe-inspiring needs to extend a denoising approach for the noise removal from degraded satellite images. For satellite images, researchers developed extensive diversity of denoising systems. Although, the improvement of a capable 
denoising algorithm maintains an eye on the conservation of very well details is still a demanding task [4].

In the edges, the appropriate part of information lies in the image in the majority of the satellite images. Unfortunately, for image processing due to the non-stimulating mechanism's performance is exploited, the edge associated information is inclined to demean [5]. The most important objective of image denoising is to get rid of noise whilst preserve the edges and other geometrical image features. The majority of existing image denoising approaches [13] [14] use a wavelet domain method to represents and satellite image processing. The wavelet domain algorithms demonstrate outstanding consequences while they are exploited to the processing signals comprising pointwise singularities. However, for multidimensional signals, additional kind of singularities might or could not be in attendance and wavelets are not capable to knob them efficiently. This means wavelets able to detain merely restricted.

Satellite imaging is perhaps the simple image source for profitable companies and governments to formulate a resolution for the prospect. For urban area planning it can be exploited by the municipalities otherwise, it may be exploited by the armed forces to assess the objective after the strike and rapidly. The image quality influences the results in a high-quality or low-quality manner. Regrettably, all images qualities collected from the satellites are not always high-quality due to the bad sensor; heat generated electrons, vibration, incorrect ISO settings, and the clouds [1]. There are numerous options exploited by authorities to eradicate the noise in satellite image whilst observance superior features [2]. For natural life saving or target detection functions maintaining the superior features of the noisy image at some point in the denoising process is vital for people works utilizing minute particulars like the segmentation process. Bilateral Filter is considered as effectual denoising methods eliminate the surplus noise whilst conserving the edges, and this approach, gets 2 parameters from the user. The user should choose the majority appropriate parameter values to attain the mainly significant outcome.

The main aim of this paper is to model the adaptive filter to denoise by exploiting the type 2 fuzzy system in a satellite image. The developed hybrid filter comprises 3 significant phases, noise correction, noise identification, and enhancement of the image. Initially, the noisy pixels are recognized exploiting type 2 fuzzy systems. Then, the recognized noisy pixels are rectified using the ANLMF. Subsequently, the improved Pigeon Optimization-based algorithm is used to the original image straightly to improve the image intensity.

\section{Literature Review}

In 2019, Anju Asokan and J. Anitha [1], developed an optimized bilateral filter to preserve the edges and image denoising. It was exploited by diverse nature-inspired optimization approaches which able to efficiently denoising the image exclusive of blurring the edges in the image. By a bilateral filter, the image denoising needs the resolution of control parameters hence noise was eradicated and the edge detail was preserved. It was done with the aid of optimization approaches namely PSO, CS, and ACS, parameters of control in the bilateral filter were determined for best performance.

In 2019, Jie Zhanga et al [2], worked on a reconstruction of a maximum quality image from a maximum noise astronomical image and high resolution. Initially, a 2G BDCS was developed based on the benefit of CS in image denoising. Subsequently, the IBT-BTCS approach on the basis of the BDCS was developed for high noise astronomical image reconstruction and high resolution. Initially, an iterative bandelet thresholding approach was modeled to attain the best estimate of the original image. Next, to additional enhance the reconstruction of the image quality; the GSTV-SC approach was developed to alter the rebuild astronomical image in each iteration.

In 2019, S. Jayanthi and C. Vennila [3], developed the 3D-based Adaptive Multi-Resolution supervised approach which mechanically categorizes diverse areas of space-time recognition of remote images. Initially, a 3D multi-volatile remote sensor central and multispectral was modeled to accumulate data structure. Next, the 3D multi-resolution model using receipt in decontaminated parameters follows objectives on generates 3D region techniques. Associated parameters were supervised to estimate the 3D compatible multi-resolution project performance such as spectral mean relative error, root position for quadratic error, SSIM, and the correlation coefficient.

In 2020, A. Brooka et al [4], worked on CNN, a multiscale full connected CNN is modeled for the pan-sharpening of Sentinel-2A images using UAV images. The data reconstruction was evaluated using the self-governing in-situ spectral measurements and multispectral UAV images. Plant responses temporal assessment was offered by the reconstructed Sentinel-2A images exploiting chosen vegetation indices. The developed method was examined on plant measurements and also in-vivo, using assessment of water conductivity and water employ competence indexes from isotopic traits and anatomical recorded.

In 2018, Anju Unnikrishnan et al [5], worked on multispectral remote sensing information. The NDVI was an introverted parameter to detect the landcover using red and NIR information for the 
electromagnetic spectrum. NDVI was exploited to break down remote recognizing the images and review the attendance or nonattendance for the live green vegetation. The analysis was experimented in a novel publically obtainable SAT-four dataset, whereas classes included were kinds of vegetation.

\section{Developed an Adaptive Filter with the Optimization Algorithm for the Enhancement of Satellite Image}

In this section, the model of the adaptive filter to denoise the satellite image is presented. Here, the adaptive filter is designed for the noise rectification and enhancement of the image, using the aid of developed Improved Pigeon optimization Approach, and fig. 1 exhibit the schematic diagram for the similar.

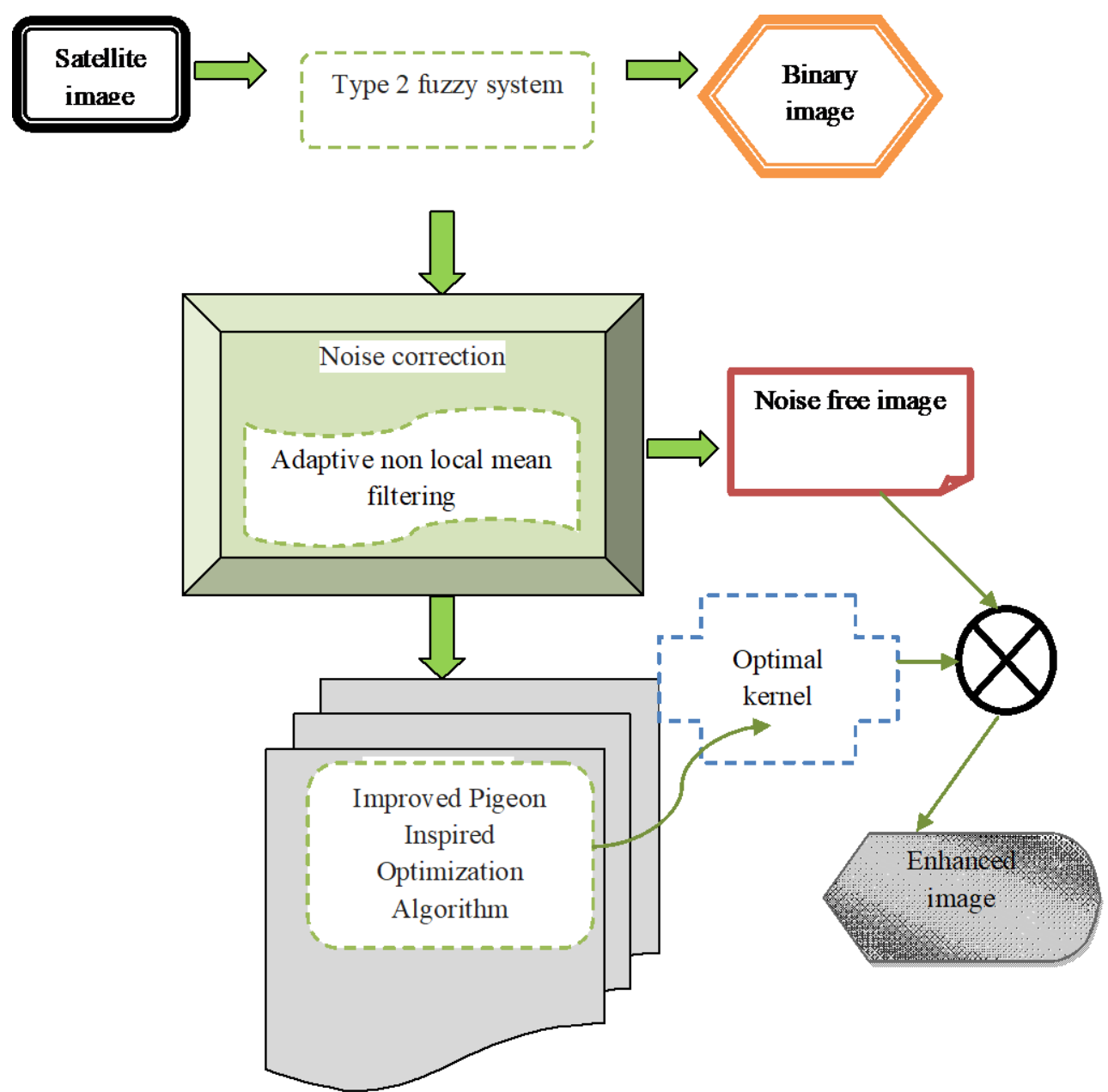

Fig. 1. Schematic diagram of the developed approach for improving the satellite image intensity

In Fig. 1, the developed adaptive filter carries out the image denoising in three phases, like identification of noise, correction of noise, and enhancement of the image. The identification of noise is performed exploiting the Type 2 fuzzy filter that transforms the input image to the binary image and cautiously identifies the pixels of noise. Subsequently, the correction of noise is performed using the ANLMF. Afterward, the kernel-based interpolation model performs the enhancement of an image using the noise-free image from ANLMF. The best for the image enhancement kernel is chosen using the optimization process, and for this reason, Improved Pigeon optimization method has been developed.

\subsection{Type 2 Fuzzy System for the Identification of Noise Identification}

In the image denoising, the primary phase is the identification of noise and it is performed by exploiting the Type 2 fuzzy filter [6]. Let us $\mathrm{I}_{\mathrm{M} \times \mathrm{N}}$ represent the input image using the size of $\mathrm{M} \times \mathrm{N}$. For identification of noise, the input image is preceded through the Type 2 fuzzy filter. After the identification procedure, the filter transforms the input image, $\mathrm{I}_{\mathrm{M} \times \mathrm{N}}$ indicates the binary image, $\mathrm{B}_{\mathrm{M} \times \mathrm{N}}$, in that 0 indicates the noise-free pixel, and 1, indicates the noisy pixel. From the type 2 fuzzy filters, the binary image is indicated in eq. (1). 


$$
\mathrm{B}_{\mathrm{M} \times \mathrm{N}}=\left\{\begin{array}{ll}
1 ; & \text { Noisy pixel } \\
0 ; & \text { Noise free pixel }
\end{array}\right\}
$$

\subsection{Adaptive Non-Local Means Filter for Rectification of Noise}

In the image, after the identification of the noisy pixels, the binary image is progressed via the ANLMF [7] for the correction of noise. The ANLMF filter performs the correction of noise procedure in the subsequent steps:

a) To the ANLMF filter, $\mathrm{B}_{\mathrm{M} \times \mathrm{N}}$ is represented as the input of the binary image.

b) Eq. (2) indicates the computation model of the local scale for the image.

$$
\mathrm{L}_{\mathrm{ij}}=\underset{\mathrm{r} \in \mathrm{Z}, \mathrm{r}>\mathrm{q}_{\mathrm{s}}}{\arg \max }\left\{\mathrm{Y}_{\mathrm{r}}(\mathrm{i}, \mathrm{j}) \geq \mathrm{Q}_{\mathrm{s}}\right\}
$$

In eq. (2), $Y_{r}(i, j)$ denotes the uniformity pixel value of $(i, j)$ and $Q_{s}$ denotes the parameter of tolerance.

c) At present, for scaling operation, the average of the pixel is computed based on the Non-Local Means Filter (NLMF) by improving the adaptive size exploited in the step (b).

d) Output the $\mathrm{E}_{\mathrm{M} \times \mathrm{N}}$ noise-free image.

\subsection{Enhancement of Image Exploiting Kernel Interpolation with the Developed Optimization Approach}

In image denoising, the final stage is the enhancement of the image, and it is performed using the developed kernel interpolation method. From the ANLMF, the noise-free image is improved using the interpolation it using the kernel which is optimally chosen via the improved Jaya pigeon optimization algorithm.

(i) Kernel Interpolation method

A kernel function $\mathrm{K}$ of size $\mathrm{k}_{1} \times \mathrm{k}_{2}$ is optimally established and the combined with $\mathrm{E}_{\mathrm{M} \times \mathrm{N}}$ for the kernel interpolation. The kernel interpolation is represented in eq. (3).

$$
\mathrm{D}_{\mathrm{M} \times \mathrm{N}}=\mathrm{E}_{\mathrm{M} \times \mathrm{N}} \otimes \mathrm{K}
$$

In eq. (3), $\mathrm{D}_{\mathrm{M} \times \mathrm{N}}$ indicates the concluding denoised image / improved image and $\otimes$ indicates the interpolation operator. It can be shown that after the image denoising, the image size remnants unchanged. The elements of the kernel function $\mathrm{K}$ state chosen to exploit the optimization process.

\section{Improved Pigeon-Inspired Optimization}

In this section, the enhanced PIO method is developed to determine optimal kernel function hence that the kernel interpolation is flourishing. The proposed method can be considered as an improved version of the existing Jaya optimization algorithm [11]. In the existing Pigeon optimization approach, the optimization is performed using the external parameters, and therefore, the approach has negligible influence from the external parameters. Using such type of algorithms for the kernel interpolation can make the image enhancement to be more accurate.

The PIO is a new intelligent learning algorithm that emulates the pigeons homing behavior. During exploiting the map and scope operator and the landmark operator, the Pigeon Inspired Optimization approach can discover the global best solutions. Additionally, the PSO is a traditional and a competent meta-heuristic learning approach. Enthused by hybrid DA [12], the PSO algorithm was introduced into the Pigeon Inspired Optimization approach and develop a new Enhanced PIO learning method to enhance optimization skills and global searching capability. The procedure of the Improved Pigeon Inspired Optimization learning approach is stated below:

Algorithm 1 shows the pseudo-code of the proposed approach.

Initialize the parameters of PSO and develop the adaptive mutation operator into PSO. In PSO, particles are initialized during each interaction using a definite probability.

The adaptive mutation operator has the ability to improve the population diversity of PSO and develop the searching space of particles. In PSO, updating equation of location, and velocity, is exhibited as follows:

$$
\begin{aligned}
& U_{i}(t+1)=\omega \cdot U_{i}(t)+1_{1} \cdot m_{1} \cdot\left(P_{i}(t)-Y_{i}(t)\right)+1_{2} \cdot m_{2} \cdot\left(P_{g b}(t)-Y_{i}(t)\right) \\
& Y_{i}(t+1)=Y_{i}(t)+U_{i}(t)
\end{aligned}
$$

In eq. (4), $\mathrm{Y}_{\mathrm{i}}(\mathrm{t})$ and $\mathrm{U}_{\mathrm{i}}(\mathrm{t})$ indicate position and the velocity of the $\mathrm{i}^{\text {th }}$ particle in $\mathrm{t}^{\text {th }}$ iteration. $\mathrm{l}_{1}$ and $1_{2}$ indicate the acceleration factors in PSO, $\omega$ represents the inertia weight, and the values of $1_{1}$ and $1_{2}$ are 
always higher than 0. $\mathrm{P}_{\mathrm{gb}}$ represents the global optimal location and $\mathrm{P}_{\mathrm{i}}$ represents the individual optimal location. $\mathrm{rn}_{1}$ and $\mathrm{rn}_{2}$ indicates the 2 arbitrary numbers among 0 and 1 .

PIO algorithm parameters are initialized and initiate the global optimal PSO values to the PIO approach. For optimization, if the fitness of the global optimal PSO values is superior to the primary PIO values, the global optimal PSO values can be transported to the PIO approach.

For parameter optimization PIO is used. The inertia weight is augmented to the PIO approach. In PIO, the inertia weight has the ability to produce a balance among local search and global search. The formulation for the inertia weight is stated in eq. (6).

$$
w(t)=w_{s}-\left(w_{s}-w_{e}\right) \times t / T
$$

In eq. (6), $w(t)$ indicates inertia weight in the $t^{\text {th }}$ iteration. When the iteration stops and $w_{s}$ indicates the initial inertia weight. $\mathrm{T}$ indicates the utmost number of iterations.

The PIO method comprises two important operators the map and landmark operator and the compass operator. In the compass and map operator, the updating formula of velocity and location are shown as follows:

$$
\begin{aligned}
& U_{i}(t+1)=w(t) \cdot U_{i}(t) \cdot e^{-R t}+m_{3} \cdot\left(Y_{g}-Y_{i}(t)\right) \\
& Y_{j}(t+1)=Y_{j}(t)+U_{j}(t+1)
\end{aligned}
$$

In eq. (8), $Y_{j}(t)$ and $U_{j}(t)$ indicates the location and the velocity of $j^{\text {th }}$ pigeon in $t^{\text {th }}$ iteration. $R$ states the compass and map factor, whose value is among 0 and $1 . \mathrm{rn}_{1}$ indicates an arbitrary number among 0 and 1.

While the compass and the map operator obtained the utmost number $\left(T_{1}\right)$, the landmark operator initiates to discover an optimum solution. The formulation for updating the pigeons' position in the landmark operator is stated as below:

$$
\begin{aligned}
& \mathrm{N}_{\text {landmark }}(\mathrm{t}+1)=\frac{\mathrm{N}_{\text {landmark }}(\mathrm{t})}{2} \\
& \mathrm{Y}_{\text {cen }}(\mathrm{t})=\frac{\sum \mathrm{Y}_{\mathrm{j}}(\mathrm{t}) \cdot \mathrm{g}\left(\mathrm{Y}_{\mathrm{j}}(\mathrm{t})\right)}{\mathrm{N} \cdot \sum_{\mathrm{g}}\left(\mathrm{Y}_{\mathrm{j}}(\mathrm{t})\right)} \\
& \mathrm{Y}_{\mathrm{j}}(\mathrm{t}+1)=\mathrm{Y}_{\mathrm{j}}(\mathrm{t})+\mathrm{rn}_{4} \cdot\left(\mathrm{Y}_{\text {cen }}(\mathrm{t}+1)-\mathrm{Y}_{\mathrm{j}}(\mathrm{t})\right)
\end{aligned}
$$

In the landmark operator procedure, the amount of pigeons minimizes through partially in each iteration. $\mathrm{N}_{\text {landmark }}(\mathrm{t})$ indicates the number of pigeons in $\mathrm{t}^{\text {th }}$ iteration. $\mathrm{rn}_{4}$ indicates an arbitrary number among 0 and $1, \mathrm{Y}_{\text {cen }}$ indicates the center locations of pigeons, and $\mathrm{g}($.$) indicates the fitness model.$

While the PIO approach obtains the utmost quantity of landmark operator $\mathrm{T}_{2}$ the PIO approach halts, and the position of global best solution, $\mathrm{Y}_{\mathrm{gb}}$ indicates the ultimate results of parameter optimization.

\footnotetext{
Algorithm 1: Pseudocode of proposed Improved Pigeon Inspired Optimization Algorithm

To find the global optimum solution $\mathrm{P}_{\mathrm{gb}}$, use PSO with the adaptive mutation operator by updating the position and velocity based on eq. (4)

Initialize the parameters of PIO with an arbitrary position and velocity

Update the initial position with a superior fitness value and evaluate the fitness value $P_{g b}$
}

While $\left(t>T_{1}\right)$ do

For every individual

End for

Update the position and location based on eq. (8) and (9)

Update the location of the global optimal location solution $\mathrm{Y}_{\mathrm{gb}}$

end while

While $\left(\mathrm{T}_{1}<\mathrm{t} \leq \mathrm{T}_{1}\right)$ do

For each individual

End for

Update the position using eq. (10) to (12)

Update the position $\mathrm{Y}_{\mathrm{gb}}$

End while

Return $\mathrm{Y}_{\mathrm{gb}}$ 


\section{Results and Discussions}

\subsection{Experimental Procedure}

In this section, the outcomes of the developed adaptive filter which exploits the Improved Pigeon Inspired optimization. The outcomes were estimated by taking into consideration of the several noise samples on the hyperspectral images in the database. For the experimentation of the developed adaptive filter and other conventional methods, this paper has uses measures, such as SSIM, PSNR, and SDME. The consequences of the developed adaptive filter by means of the Improved Pigeon Inspired optimization was analyzed with the various approaches, namely CS + WF [10], WF [9], and GA + Type 2 fuzzy filter [8]. The analysis methods considered for this paper are described in the next section.

\subsection{Performance Analysis}

The analysis for the utmost outcomes attained using the database for all the noise types using the developed adaptive filter beside the other approaches is shown in Fig 2, 3, and 4. Figure 2,3 and 4 shows the graphical analysis based on PSNR, SDME, and SSIM for several noise densities. Hence, it is evident that the developed adaptive filter using the Improved Pigeon optimization method is appropriate for the improvement of image for the satellite images, even for images under the influence of several noises from the analysis.

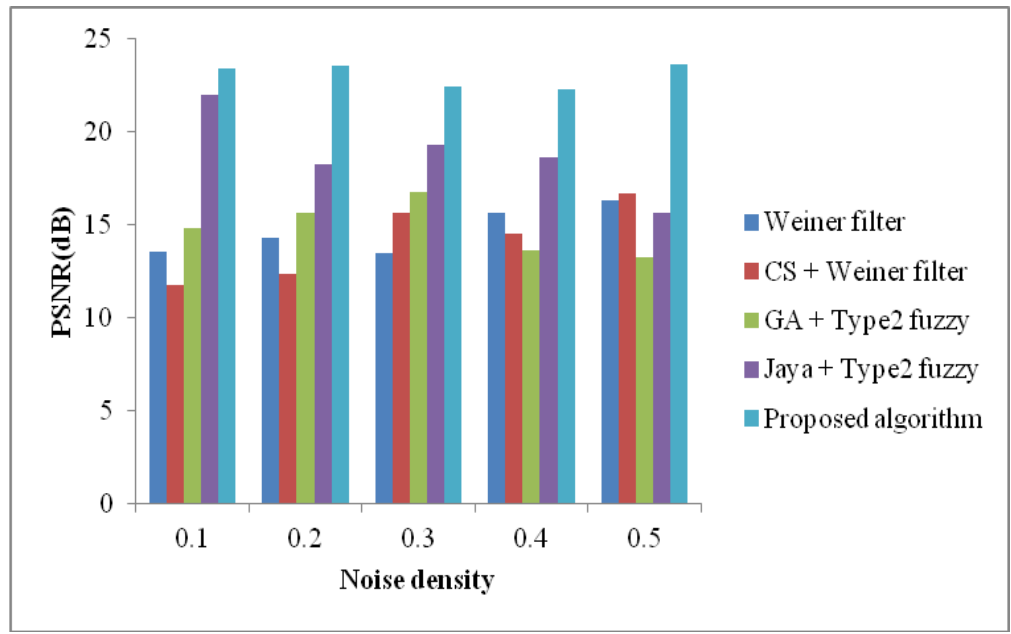

Fig. 2. Analysis of developed method regarding PSNR

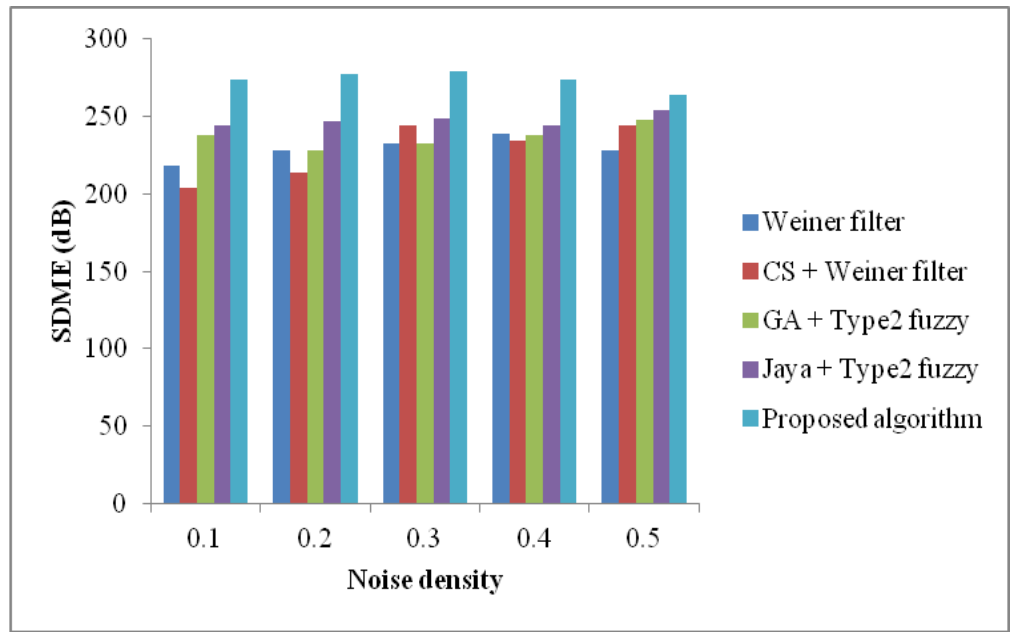

Fig. 3. Analysis of developed method regarding SDME 


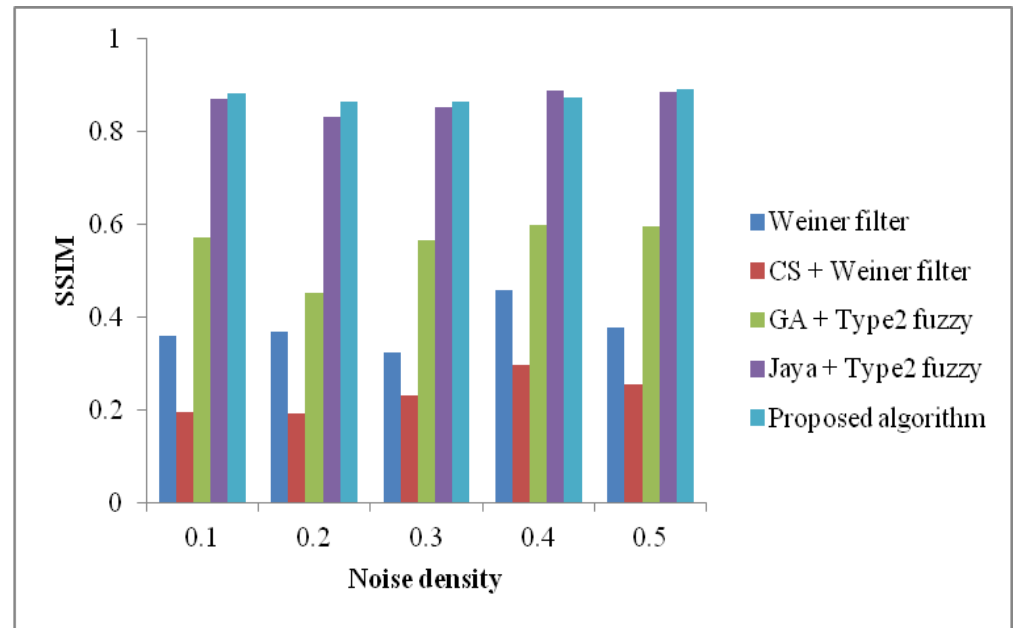

Fig. 4. Analysis of developed method regarding SSIM

\section{Conclusion}

This work proposes the satellite image denoising method to improve satellite imaging. For the enhancement of the image, this paper had developed an adaptive filter by means of a new optimization approach. The complete enhancement of the image process was performed in three phases, like noise correction, noise identification, and enhancement of the image. The developed model exploits the type 2 fuzzy filter in order to recognize the noisy pixels in the image that are rectified using ANLMF. This paper developed the Improved Pigeon optimization algorithm to enhance the image via the kernel interpolation method. From one standard database, hyper-spectral satellite images were used and assessed based on measures, like SDME, PSNR, and SSIM for the experimentation. Generally, the optimal performance was attained using the developed adaptive filter by means of the PSNR, SSIM, and SDME.

\section{References}

[1] Anju Asokan, J. Anitha,"Adaptive Cuckoo Search based optimal bilateral filtering for denoising of satellite images", ISA TransactionsIn press, corrected proofAvailable online 7 November 2019

[2] Jie Zhang, Huanlong Zhang, Xiaoping Shi, Shengtao Geng,"High noise astronomical image denoising via 2Gbandelet denoising compressed sensing" Optik, Volume 184May 2019Pages 377-388

[3] S. Jayanthi, C. Vennila,"Performance improvement in satellite image classification using adaptive supervised multi-resolution approach", Computer Communications,Volume 15015 January 2020Pages 200-208

[4] A. Brook, V. De Micco, G. Battipaglia, A. Erbaggio, A. Bonfante,"A smart multiple spatial and temporal resolution system to support precision agriculture from satellite images: Proof of concept on Aglianico vineyard", Remote Sensing of EnvironmentVolume 240April 2020.

[5] Anju Unnikrishnan, Sowmya V, Soman K P,"Deep AlexNet with Reduced Number of Trainable Parameters for Satellite Image Classification", Procedia Computer Science,Volume 1432018, Pages 931-938.

[6] A. Taskin and T. Kumbasar, "An Open Source Matlab/Simulink Toolbox for Interval Type-2 Fuzzy Logic Systems,” IEEE Symp. Ser. Comput. Intell., page no. 1561-1568, 2015.

[7] X. Lan, H. Shen, and L. Zhang, "An adaptive non-local means filter based on region homogeneity," Proc. - 2013 7th Int. Conf. Image Graph. ICIG 2013, vol. 2, no. 3, pp. 50-54, 2013.

[8] E. Kugu, "Satellite image denoising using Bilateral Filter with SPEA2 optimized parameters," RAST 2013 Proc. 6th Int. Conf. Recent Adv. Sp. Technol., pp. 217-223, 2013.

[9] S. Suresh and S. Lal, "Two-Dimensional CS Adaptive FIR Wiener Filtering Algorithm for the Denoising of Satellite Images," IEEE J. Sel. Top. Appl. Earth Obs. Remote Sens., volume. 10, number. 12, page no. 52455257, 2017.

[10] S. Suresh, S. Lal, C. Chen, and T. Celik, "Multispectral Satellite Image Denoising via Adaptive Cuckoo SearchBased Wiener Filter," IEEE Trans. Geosci. Remote Sens., pp. 1-12, 2018.

[11] D.F. Zhang, H.B. Duan, Social-class pigeon-inspired optimization and time stamp segmentation for multi-UAV cooperative path planning, Neurocomputing, vol. no 313, page no:229-246, 2018.

[12] R. Ahila, V. Sadasivam, K. Manimala, An integrated PSO for parameter determination and feature selection of ELM and its application in classification of power system disturbances, Applied Soft Computing vol.32,page no.23-37, 2015.

[13] Vinusha S,"Secret Image Sharing and Steganography Using Haar Wavelet Transform",Multimedia Research, Volume 2, Issue 2, April 2019.

[14] Nipanikar S I,Hima Deepthi V,Enhanced Whale Optimization Algorithm and Wavelet Transform for Image Steganography,Multimedia Research, Volume 2, Issue 3, July 2019. 